\title{
Using Positive Images to Manage Resistance-to-care and Combative Behaviors in Nursing Home Residents with Dementia: A Pilot Study
}

\begin{abstract}
This pilot study attempted to reduce resistance-to-care (RTC) and combative behaviors in nursing home residents with dementia by eliciting their positive affect. Four female residents with dementia were recruited from a nursing facility. Each resident was involved in one intervention trial and one control trial. During the dressing/ toileting care, when the residents manifested RTC and combative behaviors, a selected image was provided to elicit their positive affect in the intervention trial. In contrast, a blank image was provided in the control trial. The response of the residents was assessed by the Agitated Behavior Scale and the Observational Measurement of Engagement Tool. The distress level of the certified nursing assistants (CNAs) delivering the care was reported through the Distress Thermometer. Results showed that the residents displayed fewer behavioral symptoms in the intervention trial than in the control trial. The CNAs reported less distress in the intervention trial than in the control trial. These preliminary findings suggest that it might be feasible to use positive images to reduce residents' behavioral symptoms and decrease the distress of CNAs.
\end{abstract}

Key words: aggression; dementia; positive affect, positive emotion; nonpharmacological interventions; emotion regulation; resistance-to-care; combative 


\section{Introduction}

Resistance-to-care (RTC) and combative behaviors (such as squeezing, kicking and slapping) are common in nursing home residents with dementia, especially during personal care. ${ }^{1,2}$ Following the idea proposed by Volicer, ${ }^{3}$ we choose to use the term "combative" instead of "aggressive", although the language used in the cited papers was retained for the accuracy of reporting. Lack of understanding and depression have been identified as important factors in the development of RTC and combative behaviors. $^{4}$ A recent study reported that nearly $20 \%$ of certified nursing assistants (CNAs) experienced such incidents more than 10 times in a investigated month when caring for persons with dementia. ${ }^{2}$ The CNAs seem to perceive and accept violence as part of normal work. ${ }^{5}$ They reported feelings of aggression, astonishment, insult, insufficiency, powerlessness in response to the situation, and antipathy against the resident. $^{6}$

Most of the studies of non-pharmacological intervention (NPI) focus on feeding and bathing activities and suggest that using music during mealtime and bathtime may be helpful in reducing the RTC and combative behaviors. ${ }^{7}$ Despite the fact that more incidents (near to 50\%) happen during dressing and toileting care, ${ }^{2}$ few NPIs have targeted these stimuli. Considering that RTC and combative behaviors could jeopardize the nursing quality and the nursing staff's job satisfaction, it is important to develop effective and easy-to-use interventions to support CNAs in the management of such behaviors. 
The link between aggression and negative affect has been well-established in the literature. ${ }^{8,9}$ Negative affects, such as depression, boredom, loneliness, and anxiety, are commonly manifested by nursing home residents with dementia. ${ }^{10-13}$ Failure to modulate these difficult emotional experiences may lead to aggressive behavior. ${ }^{14}$ Automatic activation of positive emotions can facilitate emotion regulation and coping when an individual experiences negative feelings. ${ }^{15}$ However, persons with dementia have been reported to be deficient in emotion regulation. ${ }^{16}$ This deficiency potentially contributes to difficulties in managing negative emotions, which might lead to combative behaviors. On the other hand, it has been found that persons with dementia still have the ability to passively experience positive emotion. Activities, such as dancing, exercising, and singing, can trigger a positive mood. ${ }^{17-19}$ Cohen-Mansfield and colleagues found nursing home residents with dementia to be less aggressive while experiencing positive affects. ${ }^{20}$ Taken together, these findings support the idea that deliberately eliciting residents' positive affect might activate their emotion regulation and thereby lessen their combative behavior.

This pilot study aimed to test if deliberately eliciting positive affect in nursing home residents with dementia could decrease their behavioral problems during dressing/ toileting activities. We chose to use the images from the International Affective Pictures System (IAPS) as the emotion-eliciting stimuli, since IAPS has been used in many studies to elicit emotion. ${ }^{21}$ The IAPS is a database of images that has been validated to generate a range of emotions in individuals. ${ }^{22}$ 


\section{Methods}

\section{Participants}

Four residents with dementia were recruited from a nursing facility to participate in this study. The nursing providers at this facility helped the research team identify the residents with dementia who were frequently reported to display agitated behaviors. Any resident with a diagnosis of bipolar disorder or schizophrenia was excluded. Contact information for 19 residents' representatives was received, and 18 gave permission for their family member with dementia to participate in the study. After interviewing the main care providers of these residents, it was determined that only four residents manifested RTC and combative behaviors during dressing/toileting activities.

\section{Emotion-eliciting Stimuli}

Images selected from the IAPS were used in this study as the emotion-eliciting stimuli to elicit positive emotion. Based on the normative data (arousal and valence, both rated on a 9-point scale, with 9 referring to the highest rating and 1 referring to the lowest rating) that were provided in its technical report, ${ }^{22} 15$ positive pictures were chosen for this study. The average arousal rating of these pictures was $4.94 \pm 1.28$, and the average valence rating of these pictures was $7.44 \pm 1.60$. The themes of these images are: a baby, cliff divers, a couple, a cow, fireworks, a hiker, two nature scenes, puppies, a roller coaster, the sea, a seal, a skier, three men, and a violin. Before the experiment, a preliminary study was conducted to test each resident's response toward these images. The image to which the resident showed the most positive response was used in this resident's intervention trial. Three residents showed the most positive response to the 
baby, with the fourth resident preferring the puppies. To avoid the possibility that the residents displayed less behavioral symptoms just through having received a stimulus from the nursing staff, a control trial was built into the design of this study. A blank piece of photographic paper served as the stimulus for the control trial since it resembled the shape and weight of the stimulus used in interventional trial but without the content to elicit an emotional response.

\section{Measures}

The Agitated Behavior Scale (ABS) ${ }^{23}$ the Observational Measurement of Engagement Tool (OME), ${ }^{24}$ and the Distress Thermometer (DT $)^{25}$ were used in this study. In order to gather additional information that was not captured in the quantitative assessments, qualitative data was also collected by note-taking through direct observation.

ABS was used in this study to record the RTC and combative behaviors exhibited. The ABS is a 14-item scale developed to monitor agitation. Each item was measured on a 4point scale ranging from 1 (behavior absent) to 4 (behavior present in an extreme degree). The total potential score of ABS ranges from 14-56 with a clinical consensus that a score of 22-28 indicates mild occurrence, 29-35 indicates moderate occurrence, and more than 35 indicates severe occurrence of agitation. ${ }^{26}$ This scale has demonstrated a high degree of concurrent and construct validity, inter-rater reliability, and internal consistency by previous studies..$^{27,28}$ 
OME was used to note the participants' responses toward the stimuli. The OME was developed to record the response of individuals with dementia to stimuli and has shown a high degree of validity, inter-rater agreement (84\%), and intraclass correlation (0.78) for engagement outcome measures. ${ }^{24}$ This study used two items (attention and attitude) from the original OME. Both of these items were measured on a 7-point scale ranging from 1 (very disruptive/ very negative) to 7 (very attentive/ very positive).

DT was used in this study to assess the distress level of CNAs in relation to providing care to the four residents in this study. The DT is a self-assessment scale with scores from 0 (no distress) to 10 (extreme distress). The DT has been found valid in the screening of distressed patients with cancer and their caregivers. ${ }^{29,30}$ Each CNA was asked to report her degree of distress after each experimental trial.

\section{Procedures}

This was a crossover study. Each resident was involved in a preliminary study and two experimental trials composed of one intervention trial and one control trial. During the preliminary study, the researchers visited residents individually and presented them with the 15 pictures. The picture that the resident showed the most positive response to (assessed via OME) was used in this resident's intervention trial. During the experimental trials, one researcher accompanied the CNA while delivering dressing/toileting care to the resident. If the resident was cooperative, no experimental trial was performed. However, if the resident resisted care and exhibited combative behaviors, an intervention trial or a control trial was then performed. The researcher 
followed each dressing/toileting activity of the resident until the two trials were completed. During the intervention/control trials, after the resident resisted care and exhibited combative behavior, the CNA showed the stimulus to the resident. The CNA was encouraged to begin a short conversation with the resident (e.g., "Look at this!") and resumed providing personal care. The resident was allowed to continually hold the stimulus if desired. However, during the process of personal care, there were times when the CNA needed to take the stimulus away from the participant (e.g., transferring). After the task was completed, the CNA was encouraged to return the stimulus to the participant. The researcher, who remained unobtrusive, observed and recorded the participant's response via the OME. Notes were also made through direct observation. After the CNA completed the task, the researcher explained the ABS to the CNA and asked her to rate the participant's behaviors before and after the stimulus was presented. The CNA was also asked to report her own distress level after the care episode using the DT. All of the experimental trials were conducted in the participants' rooms, with the doors closed and the main lights turned on. Due to the shift changed and limitation of the research schedule, two CNAs (CNA4 and CNA 5) were involved in one resident's (Ms. D) experiment. For the other residents (Ms. A, B, and C), only one CNA was involved in each resident's experiment (CNA1, CNA2, and CNA3, respectively).

\section{Statistical analysis}

Data was analyzed using descriptive statistics due to the small sample size of this study. All analyses were performed by using IBM SPSS statistics version 20. 


\title{
Results
}

\section{Demographic data}

The average age of the four residents was $94.50 \pm 5.51$ years old. All were female and Caucasian and spanned from moderate to severe stages of dementia, based on the Clinical Dementia Rating Scale. ${ }^{31}$

\begin{abstract}
ABS scores
Each resident showed a decrease in ABS scores after the intervention (Figure 1). The mean difference of $A B S$ scores before and after the intervention trials is $6.75 \pm 2.22$. Three of the four residents showed no change in ABS scores before and after the control trial. Only one resident (Ms. A) showed fewer combative behaviors after the presentation of the control stimulus. The mean difference of ABS scores before and after the control trials is $1.25 \pm 2.5$.
\end{abstract}

\section{OME scores}

The mean attentive score in the intervention trials is $5.75 \pm 0.5$, all of the residents were either "slightly attentive" or "attentive" to the stimulus during the intervention trials. In the control trials, two residents were "slightly attentive" or "attentive" to the stimulus, while two were not. The mean attentive score in the controls is $4.25 \pm 1.50$ (Figure 2, left side). The mean attitude score in the intervention trials is $6.25 \pm 0.50$, all of the residents expressed a "positive" or "very positive" attitude towards the stimulus in the intervention trials. The mean attitude score in the control trials is $3.00 \pm 0.82$, with three of the 
residents showing a "slightly negative" or "negative" attitude towards the control stimulus (Figure 2, right side).

\section{DT scores}

Figure 3 shows that the CNAs had lower distress scores after the interventional trials $($ mean $=1.13 \pm 0.85)$ compared to those after the control trials (mean $=3.5 \pm 2.38)$. All but one CNA reported less distress after the intervention trials than the control trials. One CNA reported no distress following the intervention or control trial. In the case of Ms. D, which involved two different CNAs in the two trials, the CNA involved in the intervention trial reported less distress than the CNA in the control trial.

\section{Notes from the observer}

The notes taken by the observer helped the researchers to understand more details of the experiments that could not be captured by the quantitative assessments. The following are a few examples of notes.

\section{Ms. A, intervention trial}

At 20:47, CNA1 was prepared to get Ms. A ready for bed. CNA1 needed to undress Ms. A, help her with toileting, put on her nightgown, and then transfer her into bed. CNA1 brought Ms. A to her bathroom and asked her to stand up from her wheelchair. Ms. A was able to follow the instruction. However, while CNA1 tried to remove her pants, Ms. A suddenly changed her mood. She resisted and asked loudly, "Why are you doing this? I don't like it." CNA1 explained that she was going to help her to the toilet, but Ms. A still resisted. So CNA1 let Ms. A sit on the toilet with her pants on. CNA1 then passed 
the baby picture to her and said, "Look at this." Ms. A held the picture, looked at it, smiled, and said, "She is so cute." CNA1 replied, "She is the cutest!" Then Ms. A became calm and let CNA1 remove her pants. After Ms. A finished with her toileting, CNA1 provided personal care. While CNA1 tried to put on a new incontinence pad, Ms. A resisted and said, "Oh... I don't like it." CNA1 replied, "I'm sorry. There is some water [in the pad]. I'm sorry." Then, Ms. A was cooperative. While CNA1 helped Ms. A to put on the nightgown, Ms. A asked, "What are we going to do with this picture?" CNA1 replied that she would put it on the table so Ms. A will not lose it. Ms. A seemed happy with that. Then CNA1 helped Ms. A into bed. She was cooperative and reminded CNA1 to cover her well. She said good night to CNA1 and thanked her for taking care of her needs.

\section{Ms. A, control trial}

At 20:40, CNA1 was ready to prepare Ms. A for bed. She brought Ms. A to the bathroom and began to undress her. As usual, Ms. A resisted while CNA1 tried to remove her pants. She became a bit angry, held her pants, and did not allow CNA1 to remove them. Then, CNA1 passed a blank photographic paper to Ms. A and said, "Look at this." Ms. A held the blank image with her hands, looked at it, and asked, "What is this?" "Just look at it," CNA1 replied. "I don't know what you mean," Ms. A said, a bit annoyed, but she still looked at the paper. So CNA1 removed her pants and put her on the toilet. Then, while CNA1 tried to provide hygienic care, she resisted again. So CNA1 reminded Ms. A to focus on the image. "I don't know what you mean," Ms. A said. "Just look at it," CNA1 said. Ms. A was a bit annoyed, but she still focused on the image. So CNA1 took the 
chance to finish the task. Then CNA1 helped Ms. A to put on a nightgown and go to bed.

\section{Ms. B, intervention trial}

At 20:50, Ms. B was a bit sleepy, so CNA2 brought her to the bedroom. While CNA2 undressed Ms. B, she refused and said, "No, I don't want to be cold." CNA2 explained that she would be putting on a nightgown and wouldn't be cold, but Ms. B still resisted. Then CNA2 passed a baby picture to Ms. B and said, "Look at this picture." Ms. B concentrated on looking at the picture and became calm. Then, CNA2 tried to undress Ms. B again. Ms. B let CNA2 undress her and put a nightgown on her. CNA2 asked Ms. B, "Do you like the baby?" Ms. B answered, "Yes, I do." Then, CNA2 took the picture away and transferred her to the bed. Ms. B refused initially, but later let CNA2 help her into bed. Ms. B then said some bad words toward CNA2 while she was on the bed. CNA2 once again presented the baby picture to Ms. B. The picture distracted Ms. B, and she stopped cursing. Then, CNA2 turned Ms. B to one side and started to change her incontinence pad. Ms. B became agitated and scratched CNA2 on her arm. CNA2 said, "No, don't do this. It is not allowed." Then, CNA2 started to provide hygienic care. Ms. B used the picture to slap CNA2 on her arms. CNA2 said, "No, don't hurt the baby." Then, Ms. B stopped and looked at the baby picture. While CNA2 put the night brace on her feet, she was calm and looking at the picture. CNA2 didn't try to take the picture back because she didn't want to alter the resident's mood. Ms. B continued to hold the picture and fell asleep momentarily. 


\section{Ms. B, control trial}

At 21:05, Ms. B was sitting in her wheelchair in her room. CNA2 was about to get her ready for bed. CNA2 tried to undress Ms. B, but Ms. B resisted. "No," she said. Then, CNA2 passed a piece of blank photographic paper to Ms. B. "Look at this," she said. Ms. B took the piece of blank photo paper, glanced at it, and said, "There is nothing," sounding a bit annoyed. She refused to look at it again. CNA2 then started to undress Ms. B again, but Ms. B still resisted and grabbed CNA2 on her arm. Ms. B kept resisting while CNA 2 transferred her to the bed. Then, when CNA2 tried to change her incontinence pad and provide hygienic care, Ms. B slapped CNA2 on her hands. CNA2 told Ms. B not to do that, but Ms. B didn't stop. Ms. B also punched CNA2 in her stomach. While CNA2 put the night brace on her feet, Ms. B resisted and kicked CNA2.

\section{Discussion}

This pilot study used positive images to deliberately elicit residents' positive affect while they exhibited behavioral symptoms during dressing/toileting activities. The preliminary findings suggest that it might be feasible to use positive images to reduce residents' RTC and combative behaviors. Three of the four residents became less combative after the intervention trials but remained combative during the control trails. These three residents also expressed positive attitude and focused on the stimulus in the intervention trials but expressed a negative attitude and were less attentive to the stimulus in the control trials. Ms. A followed the CNA1's instructions and kept focused on the stimulus in the control trial, which could explain the reduction of her behavioral symptoms in the control trial. The difference of her ABS score in the intervention trial is 
still larger than in the control trial, showing that Ms. A's behavior was better controlled in the intervention trial. Positive pictures have been found to be an effective extrinsic source of emotion regulation in the elderly. ${ }^{32}$ The current study suggests these theories and practices may also be applied to people with dementia.

With the exception of one CNA participant, CNAs' self-reported distress levels in the intervention trials were much lower than in the control trials. One possible explanation for this finding is that the residents manifested less RTC and combative behavior after viewing the stimulus, thus avoiding distress for the CNA. Another possible explanation is that the stimulus may have triggered the CNAs' positive affects, so they experienced less distress. CNA1 reported no distress after either the intervention trial or control trial. A possible explanation is that in both trials, Ms. A exhibited less behavioral symptoms after the stimulus was presented. As the resident became more cooperative, CNA1 expressed less distress after each trial.

Although many NPIs have been developed to manage behavioral symptoms in persons with dementia, only a few focus on RTC and combative behaviors. ${ }^{33,34}$ Music has been recommended to be applied during the mealtime and bathing care. ${ }^{7}$ A person-centered care approach employed during bathing care has demonstrated a significant reduction in aggression. ${ }^{7}$ Conflicting results of the success of the ability-focused approach have been reported by different studies. ${ }^{7}$ Despite nearly half of RTC and combative behaviors happening during dressing/toileting care, ${ }^{2}$ the research focusing on this area is lacking. This pilot study presented the process of using positive images to manage the 
residents' combative behaviors during dressing/toileting activities. This intervention is feasible, as any nursing staff member could easily introduce it with little training. This intervention does not require extra time to apply, which is an advantage since the time constraints have been identified as a major barrier to NPI delivery. ${ }^{35}$ The nursing staff can decide to apply it alone or to combine it with other NPIs, depending on the clinical situation and the best interests of the resident. Although the IAPS has been developed for use in research rather than clinical situations, similar stimuli could be developed for clinical use. Person-centered care can guide us to determine which stimulus might elicit a resident's positive affect. Making such stimuli available for the nursing staff can facilitate caregiving. These stimuli might include items which provide visual, auditory and/or tactical stimulation. Once a resident's preference has been identified, it would be practical to keep these tools in his/her nightstand so that the nursing staff can easily apply it while the resident exhibits RTC and combative behaviors.

Through the reviewing of the notes taken by the observer, we found the emotioneliciting stimuli might have played a role in facilitating positive communication between the residents and CNAs, which may also have been helpful in reducing the behavioral symptoms. Although the communication was relatively shorter than the communication likely to be held by healthy parties, we did notice that our participants with dementia shared their positive emotion with the CNAs by expressing their feelings regarding the stimuli. Based on the theory of the social sharing of emotion, ${ }^{36}$ both the sharer and the recipient increase affection for one another. We can, therefore, formulate a hypothesis that sharing positive emotion enhances the social bonds between residents and the 
CNAs. This may play a role in decreasing the residents' behavioral symptoms. Further research is needed to identify interventions to enhance positive communication between CNAs and residents with dementia.

There are several limitations to this study. The small sample size limits the generalizability of the findings. Neither the researchers nor the CNAs were blinded to the experimental interventions, which may have biased their responses and documentation. Due to the change of the shift and the schedule of the study, Ms. D's experiment involved two different CNAs in the two trials. The different approach style of the two CNAs might have affected the results. The blank image we used in the control trials might have been confusing or upsetting for some residents. Further research could test and use different images based upon the OME score generated by the resident. We did not investigate the long-term effects of these stimuli nor whether they could be used proactively to prevent arising agitation during ADL care. These questions may be answered in a future study.

In summary, this pilot study reported the process of sharing positive images and facilitating positive communication in nursing home residents with dementia to manage RTC and combative behaviors during dressing/toileting care. The preliminary findings of this project suggest that it might be feasible to use an emotion-eliciting stimulus to (1) decrease residents' aggression and (2) reduce CNAs' distress levels. This intervention is practical because it is inexpensive and easy to perform at the bedside. Future 
research should test this approach with a larger sample size and, over time, determine the long-term effect of this intervention.

\section{Acknowledgements}

The authors would like to thank the residents and their authorized representatives who agreed to participate in this project. A special thank you to the clinical staff who participated or provided help in this project as well as Randall Waszynski and Gu Li for proofreading this manuscript.

\section{References}

1. Lachs MS, Rosen T, Teresi JA, Eimicke JP, Ramirez M, Silver S, et al. Verbal and Physical Aggression Directed at Nursing Home Staff by Residents. J Gen Intern Med. 2013;28:660-7.

2. Morgan DG, Cammer A, Stewart NJ, Crossley M, D'Arcy C, Forbes DA, et al. Nursing Aide Reports of Combative Behavior by Residents With Dementia: Results from a Detailed Prospective Incident Diary. J Am Med Dir Assoc. 2012;13:220-7.

3. Volicer L. Is Your Nursing Home a Battlefield? J Am Med Dir Assoc. 2012;13:195-6.

4. Galindo-Garre F, Volicer L, Van Der Steen JT. Factors related to rejection of care and behaviors directed towards others: A longitudinal study in nursing home residents with dementia. Dement Geriatr Cogn Disord Extra. 2015;5:123-34.

5. Sandvide A, Aström S, Norberg A, Saveman B-I. Violence in institutional care for elderly people from the perspective of involved care providers. Scand J Caring Sci. 2004;18:351-7.

6. Aström S, Karlsson S, Sandvide A, Bucht G, Eisemann M, Norberg A, et al. Staff's experience of and the management of violent incidents in elderly care. Scand $J$ Caring Sci. 2004;18:410-6.

7. Konno R, Kang HS, Makimoto K. A best-evidence review of intervention studies for minimizing resistance-to-care behaviours for older adults with dementia in nursing homes. J Adv Nurs. 2014;70:2167-80. 
8. Berkowitz L. Affective Aggression: The Role of Stress, Pain, and Negative Affect. In: Donnerstein RGG, editor. Human Aggression. San Diego: Academic Press; 1998. pages 49-72.

9. Berkowitz L. On the formation and regulation of anger and aggression. A cognitive-neoassociationistic analysis. Am Psychol. 1990;45:494-503.

10. Bergdahl E, Allard P, Gustafson Y. Depression among the very old with dementia. Int Psychogeriatr. 2011;23:756-63.

11. Cohen-Mansfield J, Marx MS, Werner P. Observational data on time use and behavior problems in the nursing home. J Appl Gerontol. 1992;11:111-21.

12. Moyle W, Kellett U, Ballantyne A, Gracia N. Dementia and loneliness: An Australian perspective. J Clin Nurs. 2011;20:1445-53.

13. Porter VR, Buxton WG, Fairbanks LA, Strickland T, O'Connor SM, RosenbergThompson S, et al. Frequency and characteristics of anxiety among patients with Alzheimer's disease and related dementias. J Neuropsychiatry Clin Neurosci. 2003;15:180-6.

14. Roberton T, Daffern M, Bucks RS. Emotion regulation and aggression. Aggress Violent Behav. 2012;17:72-82.

15. Tugade MM, Fredrickson BL. Regulation of Positive Emotions: Emotion Regulation Strategies that Promote Resilience. J Happiness Stud. 2007;8:31133.

16. Goodkind MS, Gyurak A, McCarthy M, Miller BL, Levenson RW. Emotion Regulation Deficits in Frontotemporal Lobar Degeneration and Alzheimer's Disease. Psychol Aging. 2010;25:30-7.

17. Williams CL, Tappen RM. Effect of Exercise on Mood in Nursing Home Residents With Alzheimer's Disease. Am J Alzheimers Dis Other Demen. 2007;22:389-97.

18. Palo-Bengtsson L, Ekman SL. Emotional response to social dancing and walks in persons with dementia. Am J Alzheimers Dis Other Demen. 2002;17:149-53.

19. Osman SE, Tischler V, Schneider J. "Singing for the Brain": A qualitative study exploring the health and well-being benefits of singing for people with dementia and their carers. Dementia. 2014. Advance online publication. doi: $10.1177 / 1471301214556291$

20. Cohen-Mansfield J, Dakheel-Ali M, Jensen B, Marx MS, Thein K. An analysis of the relationships among engagement, agitated behavior, and affect in nursing home residents with dementia. Int Psychogeriatr. 2012;24:742-52.

21. Bradley MM, Lang PJ. The International Affective Picture System (IAPS) in the 
study of emotion and attention. In: Allen JACJJB, editor. Handbook of emotion elicitation and assessment. New York, NY, US: Oxford University Press; 2007. pages 29-46.

22. Lang PJ, Bradley MM, Cuthbert BN. International affective picture system (IAPS): Affective ratings of pictures and instruction manual. Technical Report A-8. University of Florida, Gainesville, FL. 2008

23. Corrigan JD. Development of a scale for assessment of agitation following traumatic brain injury. J Clin Exp Neuropsychol. 1989;11:261-77.

24. Cohen-Mansfield J, Dakheel-Ali M, Marx MS. Engagement in persons with dementia: The concept and its measurement. Am J Geriatr Psychiatry. 2009;17:299-307.

25. Roth AJ, Kornblith AB, Batel-Copel L, Peabody E, Scher HI, Holland JC. Rapid screening for psychologic distress in men with prostate carcinoma: A pilot study. Cancer. 1998;82:1904-8.

26. Bogner, JA. The Agitated Behavior Scale. The Center for Outcome Measurement in Brain Injury. http://www.tbims.org/combi/coglog (accessed October 30, 2015).

27. Bogner JA, Corrigan JD, Stange M, Rabold D. Reliability of the agitated behavior scale. J Head Trauma Rehabil. 1999;14:91-6.

28. Corrigan JD, Bogner JA. Factor structure of the agitated behavior scale. J Clin Exp Neuropsychol. 1994;16:386-92.

29. Bevans M, Wehrlen L, Prachenko O, Soeken K, Zabora J, Wallen GR. Distress screening in allogeneic hematopoietic stem cell (HSCT) caregivers and patients. Psychooncology. 2011;20:615-22.

30. Hawkes AL, Hughes KL, Hutchison SD, Chambers SK. Feasibility of brief psychological distress screening by a community-based telephone helpline for cancer patients and carers. BMC Cancer. 2010;10:14.

31. Morris JC. The Clinical Dementia Rating (CDR): Current version and scoring rules. Neurology. 1993;43:2412-4.

32. Jonathan $\mathrm{H}$, Sandrine $\mathrm{V}$, André $\mathrm{D}$. Using humour as an extrinsic source of emotion regulation in young and older adults. Q J Exp Psychol. 2014;67:10:1895909.

33. Vickland V, Chilko N, Draper B, Low L-F, O'Connor D, Brodaty H. Individualized guidelines for the management of aggression in dementia - Part 1: key concepts. Int Psychogeriatr. 2012;24:1112-24.

34. Catic A. Nonpharmacological management of behavioral and psychological 
symptoms of dementia in long-term care residents. Ann of Long-Term Care: Clin Care and Aging. 2015;23:23-29.

35. Janzen S, Zecevic AA, Kloseck M, Orange JB. Managing agitation using nonpharmacological interventions for seniors with dementia. Am J Alzheimers Dis Other Demen. 2013;28:524-32.

36. Rimé B. Emotion Elicits the Social Sharing of Emotion: Theory and Empirical Review. Emot Rev. 2009;1:60-85.

Figure legends

Fig. 1. Residents' ABS scores before and after the intervention trials and control trials.

Fig. 2. Resident's OME scores in the intervention trials and control trials.

Fig. 3. CNAs' DT scores after the intervention trials and control trials. 